\title{
Residual Type Schizophrenia
}

National Cancer Institute

\section{Source}

National Cancer Institute. Residual Type Schizophrenia. NCI Thesaurus. Code C35007.

A subtype of schizophrenia in which the individual has suffered an episode of schizophrenia but there are no longer any delusions, hallucinations, disorganized speech or behavior. Residual symptoms are seen in the form of negative symptoms (such as flat affect or avolition) or attenuated positive symptoms (such as odd beliefs). 